Revista PSICOLOGIA, 2015, Vol. 29 (2), 63-70. doi: http://dx.doi.org/10.17575/rpsicol.v29i2.1045

\title{
Efeito de Inibição Colaborativa: Revisão de algumas variáveis de influência
}

\author{
Magda Saraiva $^{1}$, Pedro B. Albuquerque ${ }^{1}$, Joana Arantes ${ }^{1}$ \\ ${ }^{1}$ Escola de Psicologia, Universidade do Minho.
}

\begin{abstract}
Resumo: 0 presente artigo tem como objetivo proporcionar a compreensão do efeito de inibição colaborativa. Este efeito, traduz-se no facto de o desempenho mnésico de um grupo colaborativo ser inferior ao desempenho de um grupo nominal. Para tal, ao longo desta revisão focaremos os principais estudos e resultados no âmbito da memória colaborativa; identificaremos os fatores e variáveis que influenciam este efeito; e, finalmente debateremos as principais hipóteses explicativas do efeito de inibição colaborativa.
\end{abstract}

Palavras-chave: Inibição colaborativa; Memória colaborativa; Estratégias de evocação; Grupo.

Collaborative Inhibition Effect: Review of some influent variables: This article aims to provide an understanding of collaborative inhibition effect. This effect is reflected in the fact that the memory performance of collaborative group is less than the performance of the nominal group. To this end, throughout this review we will focus on the main studies and results in the context of collaborative memory; identify the factors and variables that influence this effect; and finally we will discuss the main explanatory hypotheses of collaborative inhibition effect.

Keywords: Collaborative inhibition; Collaborative memory; Retrieval strategies; Group.

Ao longo dos últimos 20 anos temos vindo a assistir ao interesse crescente na investigação sobre a memória colaborativa. Com uma história ainda recente, a própria designação de memória colaborativa não é consensual, tal como não o é a sua definição. Neste artigo utilizaremos o termo e a definição de memória colaborativa como a recordação que envolve grupos de duas ou mais pessoas (Meade \& Roediger, 2009). Partindo desta definição de memória colaborativa, somos levados a crer que, quando um grupo recorda uma informação que experienciou ou processou em grupo, o seu desempenho é superior ao de cada membro do grupo, isto é, intuímos que "duas cabeças pensam melhor que uma". Efetivamente, os estudos de memória colaborativa têm vindo a corroborar este facto, pois a evocação de informação tem-se revelado consistentemente superior nos grupos colaborativos (de duas ou mais pessoas) quando comparada com a evocação de cada indivíduo isoladamente (e.g., Weldon \& Bellinger, 1997). Este facto é particularmente notado quando é utilizado o método aditivo (ver Lorge \& Solomon, 1955) para calcular a quantidade de informação evocada pelo grupo colaborativo. Veja-se o exemplo de um grupo colaborativo constituído pelo participante A e pelo participante B, em que o participante A evoca os itens 1, 2 e 3 (total de itens evocados $=3$ ) e o participante $B$ evoca os itens $1,2,5,6$ e 7 (total de itens evocados $=5$ ). A média da evocação individual, segundo o modelo aditivo, é de 4. No entanto, o desempenho colaborativo corresponde a 6 itens evocados $(1,2,3,4,5$, e 6$)$. Como se pode verificar, o desempenho do grupo - 6 - é superior à média do desempenho individual - 4 - para a mesma informação.

No entanto, nos estudos de memória colaborativa, o método mais comum de comparação entre o desempenho de um grupo colaborativo e o desempenho individual consiste na criação de um grupo nominal. 0 grupo nominal não corresponde a qualquer grupo formalmente constituído, pois ele é composto pelos desempenhos individuais de tantos indivíduos quantos os que constituem o grupo colaborativo. De modo a clarificar o desempenho de um grupo nominal, tomemos em consideração o exemplo de um grupo constituído por três elementos (A, B, C). Na tarefa colaborativa este grupo evoca os itens 1, 2, 3, 4 e 5 (total de itens evocados = 5). Na tarefa individual, o participante A evoca os itens 1, 2, 3, e 4 (total de itens evocados = 4), o participante B evoca os itens 2, 3, 5, e 6 (total de itens evocados = 4), e o participante $C$ evoca os itens $5,7,9$, e 10 (total de itens evocados $=4$ ). Segundo o modelo aditivo, o desempenho médio individual corresponderia a 4. No entanto, para formar o grupo nominal, são somados todos os itens evocados individualmente pelos três participantes, excluindo os que se repetem, o que

\footnotetext{
${ }^{1}$ Dados de contacto para correspondência: Magda Saraiva, Escola de Psicologia (EPsi), Campus de Gualtar, 4710-057, Braga, Portugal. E-mail: magda.saraiva@gmail.com.
} 
neste caso corresponde então à evocação dos itens 1, 2, 3, 4, 5, 6, 7, 9 e 10 (total de itens evocados = 9). Os itens repetidos são contabilizados uma única vez, visto que o objetivo do grupo nominal é representar o potencial de evocação máximo desse conjunto de pessoas. Calculada a quantidade de informação evocada pelo grupo nominal, ou seja, o potencial de evocação dos três elementos do grupo colaborativo quando evocam informação de modo individual, é possível constatar que o seu desempenho é, habitualmente, superior ao desempenho dos três membros do grupo quando recordam a mesma informação de modo colaborativo. Este é o resultado mais comum e robusto nos estudos sobre memória colaborativa, sendo designado por inibição colaborativa (Weldon \& Bellinger, 1997). Os referidos autores definiram inibição colaborativa como sendo um fenómeno em que, contraintuitivamente, um grupo composto por duas ou mais pessoas recorda significativamente menos informação quando comparado com a soma do potencial individual de recordação dessas mesmas pessoas (grupo nominal).

Mas porque ocorre este fenómeno? É replicável com diferentes tipos de estímulos? A sua magnitude varia com a dimensão do grupo? É possível eliminá-lo? E inverter a sua tendência? 0 presente artigo baseia-se na revisão da investigação feita nesta temática e tem como objetivo responder àquelas questões, percorrendo algumas das teorias explicativas e explorando alguns dos resultados mais consensuais.

\section{À Procura de uma Explicação para o Efeito de Inibição Colaborativa}

0 fenómeno de inibição colaborativa parece assemelhar-se ao efeito de bloqueio que por vezes surge associado a tarefas realizadas em grupo (para uma revisão ver Paulus, 2000). De acordo com Diehl e Stroebe (1987), por vezes a quantidade e a qualidade das ideias produzidas em grupo é inferior à soma das ideias produzidas individualmente por cada membro do grupo. De acordo com os mesmos autores, existem diversas explicações para a ocorrência do efeito de bloqueio, como seja a inibição de produção, que ocorre quando um elemento do grupo está a evocar/produzir informação ou ideias e impede que os outros membros do grupo evoquem/produzam informação nesse mesmo momento. Esta inibição de produção pode levar ao esquecimento da informação que se pretendia verbalizar ao grupo.

Estudos da inibição colaborativa revelam que o bloqueio na produção de ideias é por si só uma explicação para o fenómeno (Weldon \& Bellinger, 1997). Em tarefas de evocação colaborativa pode ser utilizada a técnica de discussão livre ${ }^{2}$, onde é pedido aos participantes que nomeiem um redator e, livremente e sem regras definidas, evoquem o máximo de informação que recordam. Esta tarefa de evocação normalmente não tem um tempo limite, terminando quando os participantes não conseguem evocar mais informaç̧ões. Uma vez que não existe uma ordem ou instruções definidas para esta evocação, não existe nenhum fator que impeça um indivíduo de evocar uma informação em qualquer momento, e com isso não há oportunidade para o bloqueio na produção de ideias. Os resultados de estudos que recorrem à técnica de discussão livre têm, no entanto, revelado a existência de inibição colaborativa (e.g., Barber, Harris, \& Rajaram, 2014; Barber \& Rajaram, 2011; Dahlström, Danielsson, Emilsson, \& Andersson, 2011; Kelley, Pentz, \& Reysen, 2014; Weldon \& Bellinger, 1997), o que retira força à hipótese de a inibição colaborativa ser explicada pelo bloqueio na produção de ideias.

Outra explicação para a ocorrência da quebra de desempenho mnésico em grupo é a inibição social. Esta proposta de Camacho e Paulus (1995) defende a existência de ansiedade inerente a qualquer interação social. Neste caso, cada membro do grupo poderia sentir-se avaliado pelos outros de cada vez que produz informação, o que inibe a geração de ideias. Do mesmo modo, vários estudos revelam que em grupo regista-se, por vezes, preguiça social ${ }^{3}$ (para uma revisão ver Garrido, 2012). A preguiça social diz respeito ao facto de, em grupo, cada indivíduo tender a reduzir o esforço que aplica na concretização de um objetivo grupal. Esta preguiça vai então resultar num menor desempenho do grupo, em comparação com a soma do desempenho individual de cada membro desse grupo (para uma revisão ver Latané, Williams, \& Harkins, 1979). No entanto, este argumento falha novamente na explicação da ocorrência da inibição colaborativa. No estudo de Weldon, Blair e Huebsch (2000) os autores pretenderam perceber se os fatores motivacionais/sociais eram responsáveis pela inibição colaborativa. Desta forma, os autores optaram por aumentar os níveis de motivação dos participantes através de incentivos monetários, forçando a evocação, aumentando a responsabilidade pessoal de cada membro do grupo e a coesão do grupo. No entanto, a inibição colaborativa fez-se notar, revelando assim que os fatores motivacionais/sociais não chegam para justificar este fenómeno.

Para além das semelhanças da inibição colaborativa com o efeito de bloqueio de desempenho em grupo, esta é, para alguns autores (e.g., Basden \& Basden, 1995; Basden, Basden, Bryner, \& Thomas III,

\footnotetext{
2 Tradução sugerida por Saraiva, Albuquerque e Arantes (2014a) para o termo em inglês "free-for-all".

${ }^{3}$ Tradução para o termo em inglês "social loafing" sugerido por Garrido (2012).
} 
1997), igual ao efeito de parte da lista como pista 4 . Segundo Slamecka (1986), o efeito de parte da lista como pista ocorre quando o indivíduo estuda uma lista de palavras e no momento da evocação tem acesso a algumas das palavras dessa lista como pista para a recordação das restantes. Os resultados deste efeito revelam de forma consistente que a evocação dos participantes sujeitos à apresentação de parte da lista é inferior à dos participantes que não têm acesso a essas palavras. É também inferior ao desempenho de participantes que apenas têm acesso a um exemplar da lista de palavras como pista (Slamecka,1986). Para Basden e Basden (1995), o pior desempenho individual na presença de pistas (quando estas são parte da informação que foi codificada e deve ser evocada) ocorre porque os indivíduos são obrigados a abandonar as suas próprias estratégias de organização e evocação da informação por estas deixarem de ser compatíveis e eficazes.

É no seguimento dos estudos do efeito de parte da lista como pista que surge a explicação mais comum para a presença de inibição colaborativa em tarefas de memória colaborativa - hipótese da interrupção das estratégias individuais de evocação (Basden et al., 1997). Segundo esta hipótese, cada indivíduo desenvolve estratégias idiossincráticas de organização da informação durante a fase de codificação. Assim, no momento da recuperação em grupo, a evocação de informação por parte de cada membro do grupo funciona como pista para os outros. Por si só isto pode alterar a organização da estratégia de evocação da informação codificada por cada um dos elementos do grupo. Neste sentido, a evocação em grupo fará com que as estratégias individuais de recuperação sejam interrompidas traduzindo-se numa quebra de desempenho. Como é constatável, parte da lista como pista e disrupção da estratégia de recuperação podem ser vistos como efeitos semelhantes, que ocorrem, respetivamente, quando um indivíduo ou os elementos de um grupo tentam recuperar informação previamente aprendida. Ao fazê-lo através de uma estratégia de recuperação organizada, esta pode falhar. Seja pela apresentação de outros itens de uma lista (parte da lista como pista), seja pela evocação dos outros elementos do grupo (disrupção das estratégias de recuperação). Como consequência destas falhas, ocorre um decréscimo no desempenho mnésico (Garcia-Marques \& Garrido, 2015; para uma revisão ver Garrido, Garcia-Marques, \& Hamilton, 2012).

Esta tornou-se a explicação mais frequente para a inibição colaborativa porque, ao contrário das explicações anteriormente referidas, tem sido suportada pelos resultados de vários estudos. Entre eles, está o facto de a inibição colaborativa ter um caráter não permanente. Ou seja, se a um participante que anteriormente fez parte de um grupo colaborativo for pedido para evocar individualmente a mesma informação, não raras vezes é recordada informação que durante a tarefa colaborativa tinha sido inibida (e.g., Basden et al., 1997; Basden, Basden, \& Henry, 2000; Blumen \& Rajaram, 2008; Rajaram \& PereiraPasarin, 2007). Outra fonte de suporte para esta hipótese explicativa para o efeito de inibição colaborativa é o facto de este efeito ser atenuado quando todos os membros do grupo utilizam estratégias de evocação similares, como iremos explorar posteriormente neste artigo.

Na secção seguinte deste artigo pretendemos discutir os resultados de alguns estudos, focando a replicação e/ou atenuação do efeito de inibição colaborativa, com base em diferentes métodos e procedimento usados.

\section{Fatores que Conduzem à Replicação do Efeito de Inibição Colaborativa}

Diversos estudos têm revelado que o efeito de inibição colaborativa ocorre com uma grande diversidade de estímulos, tarefas e tipos ou métodos de evocação, revelando-se um fenómeno replicável e com resultados robustos. Entre eles encontram-se listas de palavras frequentes e concretas (e.g., Saraiva, Albuquerque, \& Arantes, 2014c), listas de exemplares de categorias (Basden et al., 1997), listas de palavras não relacionadas (Andersson, Hitch, \& Meudell, 2006; Finlay, Hitch, \& Meudell, 2000), listas de palavras associadas (Saraiva, Albuquerque, \& Arantes 2014b; Thorley \& Dewhurst, 2007; Weigold, Russell, \& Natera, 2014), histórias (Takahashi \& Saito, 2004), imagens (Finlay, et al., 2000), eventos com intensidade emocional (Yaron-Antar \& Nachson, 2006) e vídeos (Andersson \& Rönnberg, 1995). A multiplicidade de estímulos utilizados no estudo da memória colaborativa permite perceber que, mesmo com diferenças procedimentais e metodológicas entre os diferentes estudos, o efeito de inibição colaborativa é robusto.

Alguns estudos têm tentado compreender se o efeito de inibição colaborativa é transversal a todas as idades, ou se, pelo contrário, existe uma idade crítica a partir da qual este efeito se torna mais presente. Neste sentido, destacamos os estudos de Andersson (2001) e de Ross, Spencer, Blatz e Restorick, (2008), por terem estudado este fenómeno em faixas etárias diferentes.

\footnotetext{
${ }^{4}$ Tradução do termo em inglês "part-list cueing" sugerido por Saraiva et al. (2014).
} 
O estudo de Andersson (2001) comparou o desempenho de crianças de 7 e 15 anos de idade numa tarefa de memória colaborativa. Os resultados revelaram que com pares de crianças de 7 anos, de 15 anos e pares mistos (um elemento de 7 anos e outro de 15 anos), o efeito de inibição colaborativa é observado, o que mostra que desde idades muito precoces a colaboração em tarefas de memória produz algum prejuízo no desempenho mnésico.

Por seu lado, o estudo de Ross e colaboradores (2008), comparou adultos e idosos quanto à recordação de informação estando sozinhos (grupo nominal) ou com as/os suas/seus cônjuges (grupo colaborativo). Os resultados revelaram um claro efeito de inibição colaborativa, sendo ainda de notar que os idosos apresentam um desempenho inferior aos adultos, tanto no grupo nominal como no grupo colaborativo. No seu conjunto, os resultados destes dois estudos mostram que a inibição colaborativa é um efeito que ocorre independentemente da idade dos participantes.

Em tarefas de memória colaborativa, são utilizados dois métodos principais de evocação: vez-àvez ${ }^{5}$ ou discussão livre (já descrito anteriormente). No método vez- à- vez é pedido aos participantes do grupo colaborativo que evoquem cada estímulo/informação à vez, passando de seguida a evocação a outro membro do grupo e assim sucessivamente. Isto é, neste método, cada participante do grupo colaborativo dispõe de 10 segundos (tempo máximo) para evocar uma informação, sendo que, de seguida é dada a vez de evocação ao outro membro do grupo. Sempre que um participante necessita de menos de 10 segundos para evocar cada informação, a vez passa imediatamente ao outro participante. A tarefa de evocação é dada por terminada após três tentativas de evocação falhadas. Alguns estudos têm tentado compreender se a utilização destes métodos de evocação influenciam a quantidade de informação evocada pelo grupo, e consequentemente o efeito de inibição colaborativa. Neste sentido, destacamos o estudo de Thorley e Dewhurst (2007) onde os resultados revelaram que o método de evocação, vez-à-vez ou discussão livre, não parece ter qualquer efeito na quantidade de informação corretamente evocada. No entanto, no teste de evocação individual posterior à evocação colaborativa, os resultados revelam que participantes que na fase de colaboração utilizaram o método vez-à-vez, evocam mais informação correta do que os participantes que estiveram sujeitos ao método discussão livre na fase de evocação colaborativa. Este resultado revela que o método vez-à-vez favorece a quantidade de informação evocada individualmente após uma tarefa de evocação colaborativa quando comparada com o método discussão livre.

Relativamente ao tamanho do grupo e a sua influência no efeito de inibição colaborativa, destacamos o estudo de Thorley e Dewhurst (2007) porque, apesar do seu objetivo principal ser o estudo da produção de memórias falsas em tarefas de memória colaborativa, utiliza grupos de tamanho variável (individuais, pares, trios e quartetos), comparando os seus desempenhos na evocação correta de informação. Neste sentido, foram utilizadas listas de palavras associadas que os participantes ouviam e, de seguida, evocavam o máximo de palavras que recordavam. Esta tarefa era realizada individualmente, em pares, trios ou quartetos. De salientar que metade dos participantes, na recordação em pares, trios ou quartetos, evoca informação segundo o método vez-à-vez e, outra metade, com o método discussão livre. Os resultados revelaram que independentemente do número de participantes que compõem cada grupo, o grupo nominal evocou uma quantidade superior de informação quando comparado com o grupo colaborativo. No que diz respeito ao tamanho do grupo, os resultados do referido estudo revelam que à medida que o tamanho do grupo aumenta, aumenta a quantidade de informação evocada, no entanto, o desempenho do grupo nominal supera sempre o desempenho do grupo colaborativo, denotando um claro efeito de inibição colaborativa.

\section{Fatores que atenuam ou eliminam o efeito de inibição colaborativa \\ Estudo repetido}

É sabido, da investigação sobre a memória individual, que o estudo repetido da informação aumenta a sua recordação (e.g., Braun \& Rubin, 1998). Neste sentido, Basden, Basden e Henry (2000) quiseram compreender o papel do estudo repetido na quantidade de informação evocada em grupos colaborativos, bem como o seu efeito na inibição colaborativa. Desta forma, a grupos de dois ou quatro elementos, eram apresentadas listas de exemplares de categorias, às quais se seguia uma tarefa de evocação colaborativa, sendo esta tarefa repetida três vezes. No final da evocação colaborativa repetida os participantes foram sujeitos a uma tarefa de evocação individual. Os resultados revelaram que a exposição repetida às listas de palavras e respetiva evocação repetida eliminou o efeito de inibição colaborativa. A análise dos resultados mostrou ainda que a eliminação do efeito de inibição colaborativa se ficou a dever ao aumento da quantidade de palavras evocadas pelo grupo colaborativo.

\footnotetext{
${ }^{5}$ Tradução para o termo em inglês "turn taking" sugerido por Saraiva et al. (2014a).
} 
Intervalo de retenção

Com o intuito de compreender o papel do tempo decorrido entre a fase de estudo e a fase de evocação da informação, Takahashi e Saito (2004) recorreram a um intervalo de retenção de uma semana. Assim, na fase de estudo os participantes foram expostos a uma história e, passada uma semana, evocavam o máximo de informação que recordavam da história. Esta evocação ocorria tanto em pares como individualmente. Os resultados revelaram que o intervalo de uma semana eliminou o efeito de inibição colaborativa. De salientar que numa outra experiência (Exp.1, Takahashi e Saito, 2004), os autores replicaram o efeito de inibição colaborativa como o mesmo material (história) e um intervalo de retenção mais curto. Estes resultados permitem concluir que a eliminação do efeito de inibição colaborativa se deve mesmo ao tempo decorrido entre as duas fases.

\section{Tipo de tarefa de memória}

Um fator que se tem revelado determinante na eliminação do efeito de inibição colaborativa é o tipo de tarefa de memória. Neste sentido, destacamos aqui os estudos que recorreram a tarefas de reconhecimento e a tarefas de evocação serial, uma vez que em tarefas de evocação livre o efeito em causa é muitas vezes replicado (e.g., Clark, Hori, Putman, \& Martin, 2000; Ross, Spencer, Linardatos, Lam, \& Perunovic, 2004; Saraiva, Albuquerque, \& Arantes, 2014c).

0 estudo de Clark et al., (2000) teve como objetivo principal estudar o desempenho dos grupos colaborativos (pares e trios) em tarefas de reconhecimento. Na Experiência 1 os participantes viam quatro listas de palavras e após a apresentação de cada lista de palavras seguia-se uma tarefa de reconhecimento. Nesta tarefa, cada participante assinalava individualmente numa folha de papel se a palavra tinha sido apresentada anteriormente ("velha") ou não ("nova"). De seguida, em grupo de dois ou três elementos, os participantes procuravam chegar a um consenso acerca do reconhecimento da palavra, isto é, em grupo deviam definir se uma palavra que já tinham identificado anteriormente como "nova" ou "velha" se deveria manter com esse estatuto. Esta decisão deveria ser consensual e seguia o método de discussão livre. Os resultados revelaram que em colaboração a média de acertos (reconhecimento correto) é superior quando comparada com a média de acertos dos dois ou dos três elementos do grupo antes da colaboração. Este resultado revela não apenas a possibilidade de eliminação do efeito de inibição colaborativa, como ainda a existência de facilitação colaborativa, isto é, o desempenho colaborativo supera o desempenho nominal neste tipo de tarefas. Segundo Clark e colaboradores (2000), a diferença de resultados entre tarefas de evocação e tarefas de reconhecimento relativamente ao efeito e inibição colaborativa é justificada pela hipótese da interrupção das estratégias individuais. Isto é, durante as tarefas de evocação, as palavras evocadas pelos outros membros do grupo funcionam como pistas para a evocação, o que interfere negativamente com a evocação, causando uma diminuição no desempenho do grupo colaborativo quando comparado com o desempenho do grupo nominal. No entanto, em tarefas de reconhecimento isto não acontece pois a estratégia não se baseia em pistas que permitam, ou não, a evocação correta de informação. A capacidade de reconhecimento parece estar dependente de um consenso entre elementos do grupo, ou seja, os membros do grupo precisam apenas de convencer o restante grupo de que viu ou não viu (reconhece ou não reconhece) aquele estímulo.

Relativamente a tarefas que envolvem uma evocação serial, isto é, que consistem na evocação da informação pela ordem em que esta foi anteriormente apresentada, destacamos o estudo de Saraiva et al., (2014c). Neste estudo, os autores tiveram como objetivo perceber se dotando os participantes da mesma estratégia de evocação de informação (Mnemónica dos Lugares), e recorrendo a uma tarefa de evocação serial, o efeito de inibição colaborativa era atenuado. Os resultados revelaram que o recurso a uma tarefa de evocação serial elimina o efeito de inibição colaborativa. Segundo os autores, a tarefa de evocação serial permite aos elementos do grupo codificar a informação de modo similar, o que, à semelhança da proposta de Finlay, Hitch e Meudell (2000), conduz a uma estratégia de evocação semelhante, não permitindo a interrupção das estratégias individuais de evocação e consequente eliminação do efeito de inibição colaborativa.

\section{Perícia}

Por fim, a perícia dos participantes em tarefas colaborativas revela-se uma variável capaz de eliminar o efeito de inibição colaborativa. Meade, Nokes e Morrow (2009) realizaram um estudo onde tiveram por objetivo compreender de que modo a perícia em partilhar informação afetava o efeito de inibição colaborativa. Neste sentido, na sua experiência, compararam o desempenho de pilotos experientes (instrutores de voo), pilotos pouco experientes (alunos de uma escola de aviação) e não pilotos (estudantes universitários sem ligação à aviação) numa tarefa de evocação colaborativa. Aos participantes eram apresentados cenários de avarias/problemas aéreos, sendo que, de seguida era pedido que evocassem o máximo de informação que recordavam do cenário, individual ou 
colaborativamente (pares de participantes). Os resultados revelam a replicação do efeito de inibição colaborativa tanto para os pilotos sem experiência como para os não pilotos. No entanto, o resultado mais importante diz respeito ao grupo dos pilotos experientes. Neste grupo, não só o efeito de inibição colaborativa é eliminado como ainda se verifica o efeito de facilitação colaborativa. Ou seja, neste grupo, quando a evocação de informação é feita de modo colaborativo, o desempenho é superior ao desempenho obtido na tarefa individual. Segundo os autores, este resultado pode ser explicado devido ao facto de, durante a sua formação, os pilotos serem dotados de estratégias eficazes de comunicação e partilha de informação. Assim, segundo os autores deste estudo, o facto de os pilotos experientes terem estratégias de partilha de informação semelhantes, faz com que não exista interrupção das estratégias individuais de evocação durante a evocação colaborativa, ao contrário do que ocorre com os outros dois grupos de participantes, que não partilham estratégias colaborativas. No mesmo sentido destaca-se o estudo de Garcia-Marques e colaboradores (2012). Estes autores, num estudo acerca da formação de impressões numa tarefa de memória colaborativa, concluíram que, quando os membros de um grupo colaborativo organizam a informação pela mesma ordem em que foi apresentada, o efeito de inibição colaborativa é atenuado. De igual modo, Saraiva, Albuquerque e Arantes (2015) verificaram que quando um grupo colaborativo é sujeito a uma tarefa de evocação serial, onde no momento da recuperação a informação segue a mesma organização da codificação, o efeito de inibição colaborativa é eliminado.

\section{CONCLUSÃO}

0 efeito de inibição colaborativa, presente em tarefas de memória colaborativa, tem sido amplamente replicado. Os grupos colaborativos apresentam frequentemente um desempenho inferior ao desempenho dos grupos nominais em tarefas de evocação. Este efeito tem-se revelando robusto pois, como foi possível perceber ao longo deste artigo, ele é replicável recorrendo a diferentes tipos de estímulos, tamanhos de grupos colaborativos diversos ou idades dos participantes. No entanto, verifica-se também que este efeito é passível de ser atenuado ou até mesmo eliminado, manipulando, por exemplo, algumas variáveis procedimentais como o tipo de tarefa de memória.

Neste sentido, têm sido apontadas algumas explicações para a ocorrência deste efeito. A que mais se destaca, por recolher mais suporte empírico, é a hipótese proposta por Basden e colaboradores (1997), da interrupção das estratégias individuais de recuperação. Tendo em conta esta explicação do efeito de inibição colaborativa, sugerimos que estudos futuros analisem especificamente o papel das estratégias individuais de recuperação na quantidade de informação evocada em grupo, dotando, por exemplo, os participantes com estratégias de evocação semelhantes. Por outro lado, deve ser tido em conta que o processo de codificação, nomeadamente o que diz respeito à organização da informação, assume um papel fundamental no desempenho do grupo colaborativo (e.g., Garcia-Marques et al., 2012, 2015; Meade \& Roediger, 2009; Saraiva et al., 2015), pelo que estudos futuros devem explorar aquele processo.

\section{Referências}

Andersson, J. (2001). Net effect of memory collaboration: How is collaboration affected by factors such as friendship, gender and age?. Scandinavian Journal of Psychology, 42, 367-375. doi: 10.1111/14679450.00248

Andersson, J., Hitch, G., \& Meudell, P. (2006). Effects of the timing and identity of retrieval cues in individual recall: $\mathrm{Na}$ attempt to mimic cross-cueing in collaborative recall. Memory, 14, 94-103. doi: $10.1080 / 09658210444000557$

Andersson, J., \& Rönnerberg, J. (1995). Recall suffers from collaboration: Joint recall effects of friendship and task complexity. Apllied Cognitive Psychology, 9, 199-211. doi: 10.1002/acp.2350090303

Barber, S., \& Rajaram, S. (2011a). Exploring the relationship between retrieval disruption from collaboration and recall. Memory, 19, 462-469. doi: 10.1080/09658211.2011.584389

Barber, S., Harris, C., \& Rajaram, S. (in press). Why two heads apart are better than two heads together: Multiple mechanisms underlie the collaborative inhibition effect in memory. Journal of Experimental Psychology: Learning, Memory, and Cognition. doi: 10.1037/xlm0000037

Basden, D., \& Basden, B. (1995). Some tests of the strategy disruption interpretation of part-list cuing inhibition. Journal of Experimental Psychology: Learning, Memory, and Cognition, 21, 1656-1669. doi:10.1037//0278-7393.21.6.1656

Basden, B., Basden, D., Bryner, S., \& Thomas III, R. (1997). A comparison of group and individual remembering: Does collaboration disrupt retrieval strategies?. Journal of Experimental Psychology: Learning, Memory, and Cognition, 23, 1176 -1189. doi:10.1037/0278-7393.23.5.1176

Basden, B., Basden, D., \& Henry, S. (2000). Costs and benefits of collaborative remembering. Applied Cognitive Psychology, 14, 497-507. doi: 10.1002/1099-0720(200011/12)14:6<497::AIDACP665>3.0.CO;2-4 
Blumen, H., \& Rajaram, S. (2008). Influence of re-exposure and retrieval disruption during group collaboration on later individual recall. Memory, 16, 231-244. doi: 10.1080/09658210701804495

Braun, K., \& Rubin, D. (1998). The spacing effect depends on an encoding deficit, retrieval, and time in working memory: Evidence from once-presented words. Memory, 6, 37-65. doi:10.1080/741941599

Camacho, L., \& Paulus, P. (1995). The role of social anxiousness in group brainstorming. Journal of Personality and Social Psychology, 68, 1071-1080. doi: 10.1037/0022-3514.68.6.1071

Clark, S., Hori, A., Putnam, A., \& Martin, T. (2000). Group collaboration in recognition memory. Journal of Experimental Psychology: Learning, Memory, and Cognition, 26, 1578-1588. doi: 10.1037//02787393.26.6.1578

Dahlström, Ö., Danielsson, H., Emilsson, M., \& Andersson, J. (2011). Does retrieval strategy disruption cause general and specific collaborative inhibition?. Memory, 19, 140-154. doi: 10.1080/09658211.2010.539571

Diehl, M., \& Stroebe, W. (1987). Productivity loss in brainstorming groups: Toward the solution of a riddle. Journal of Personality and Social Psychology, 53, 497-509. doi:10.1037/0022-3514.53.3.497

Finlay, F., Hitch, G., \& Meudell, P. (2000). Mutual inhibition in collaborative recall: Evidence for a retrievalbased account. Journal of Experimental Psychology, 26, 1556-1567. doi: 10.1037//02787393.26.6.1556

Garcia-Marques, L., \& Garrido, M. (2015). From idiosyncratic impressions distributed impressions of others: A case for collaborative person memory. In S. Stroessner \& J. Sherman (Eds.), Social Perception: From individuals to groups (71-89). London: Taylor \& Francis.

Garcia-Marques, L., Garrido, M., Hamilton, D., \& Ferreira, M. B. (2012). Effects of correspondence between encoding and retrieval organization in social memory. Journal of Experimental Social Psychology, 48, 200-206. doi:10.1016/j.jesp.2011.06.017

Garrido, M. (2012). Paradigma subjacente ao estudo do efeito de inibição colaborativa. Laboratório de Psicologia, 10, 251-264. doi: 10.14417/lp.674.

Garrido, M., Garcia-Marques, L., \& Hamilton, D. (2012). Hard to recall but easy to judge: Retrieval strategies in social information processing. Social Cognition, 30, 56-70. doi: 10.1521/soco.2012.30.1.56

Kelley, M., Pentz, C., \& Reysen, M. (in press). The joint influence of collaboration and part-set cueing. The Quarterly Journal of Experimental Psychology. doi: 10.1080/17470218.2014.881405

Latané, B., Williams, K., \& Harkins, S. (1979). Many hands make light work: The causes and consequences of social loafing. Journal of Personality and Social Psychology, 37, 822-832. doi:10.1037/00223514.37.6.822

Lorge, I. \& Solomon, H. (1955). Two models of group behavior in the solution of eureka-type problems. Psychometrika, 20, 139-148. doi: 10.1007/BF02288986.

Meade, M., Nokes, T., \& Morrow, D. (2009). Expertise promotes facilitation on a collaborative memory task. Memory, 17, 39-48. doi: 10.1080/09658210802524240

Meade, M., \& Roediger, H. (2009). Age differences in collaborative memory: The role of retrieval manipulations. Memory \& Cognition, 37, 962-975. doi:10.3758/MC.37.7.962

Paulus, P. (2000). Groups, teams, and creativity: The creative potential of idea- generating groups. Applied Psychology: An International Review, 49, 237-262. doi: 10.1111/1464-0597.00013

Rajaram, S., \& Pereira-Pasarin, P. (2007). Collaboration can improve individual recognition memory: Evidence from immediate and delayed tests. Psychonomic Bulletin \& Review, 14, 95-100. doi:10.3758/BF03194034

Roediger, H., \& McDermott, K. (1995). Creating false memories: Remembering words not presented in lists. Journal of Experimental Psychology: Learning, Memory, and Cognition, 21, 803-814. doi:10.1037/0278-7393.21.4.803

Ross, M., Spencer, S., Blatz, C., \& Restorick, E. (2008). Collaboration reduces the frequency of false memories in older and younger adults. Psychology and Aging, 23, 85 -92. doi:10.1037/08827974.23.1.85

Ross, M., Spencer, S., Linardatos, L., Lam, K., \& Perunovic, M. (2004). Going shopping and identifying landmarks: Does collaboration improve older people's memory?. Applied Cognitive Psychology, 18, 683-696. doi: 10.1002/acp.1023

Saraiva, M., Albuquerque, P., \& Arantes, J. (2014a). Recordar em colaboração: Custos e benefícios. Manuscrito submetido para publicação.

Saraiva, M., Albuquerque, P., \& Arantes, J. (2014b). Production of false memories in collaborative memory tasks using lists of associated words. Manuscript submitted for publication. 
Saraiva, M., Albuquerque, P., \& Arantes, J. (2015). Inhibiting the collaborative inhibition effect: The role of retrieval strategies uniformisation. Manuscript submitted for publication.

Slamecka, N. (1968). An examination of trace storage in free recall. Journal of Experimental Psychology, 76, 504-513. doi: 10.1037/h0025695

Takahashi, M., \& Saito, S. (2004). Does test delay eliminate collaborative inhibition?. Memory, 12, $722-731$. doi: $10.1080 / 09658210344000521$

Thorley, C., \& Dewhurst, S. (2007). Collaborative false recall in the DRM procedure: Effects of group size and group pressure. European Journal of Cognitive Psychology, 19, 867-881. doi: $10.1080 / 09541440600872068$

Weigold, A., Russell, E., \& Natera, S. (2014). Correction of false memory for associated word lists by collaborating groups. The American Journal of Psychology, 127, 183-190. doi: 10.5406/amerjpsyc.127.2.0183

Weldon, M., \& Bellinger, K. (1997). Collective memory: Collaborative and individual processes in remembering. Journal of Experimental Psychology, 23, 1160-1175. doi: 10.1037/02787393.23.5.1160

Weldon, M., Blair, C., \& Huebsch, P. (2000). Group remembering: Does social loafing underlie collaborative inhibition?. Journal of Experimental Psychology: Learning, Memory, and Cognition, 26, 1568-1577. doi: 10.1037/0278-7393.26.6.1568

Yaron-Antar, A., \& Nachson, I. (2006). Collaborative remembering of emotional events: The case of Rabin's assassination. Memory, 14, 46-56. doi: 10.1080/09658210444000502

Historial do artigo

Recebido 09/06/2015

Aceite $\quad 21 / 10 / 2015$

Publicado $12 / 2015$

Apoio à publicação: Fundação para a Ciência e a Tecnologia (Ministério da Educação e Ciência, Portugal) - Programa FACC 